\title{
Phased Array Antenna Analysis with the Hybrid Finite Element Method
}

\author{
Daniel T. McGrath, Member, IEEE, and Vittal P. Pyati
}

\begin{abstract}
A new analysis technique for infinite phased array antennas was developed and demonstrated. It consists of the finite element method (FEM) in combination with integral equation radiation conditions and a novel periodic boundary condition for 3-D FEM grids. Accurate modeling of rectangular, circular and circular-coaxial feeds is accomplished by enforcing continuity between the FEM solution and several waveguide modes across an aperture in the array's ground plane. The radiation condition above the array is enforced by a periodic integral equation in the form of a Floquet mode summation, thus reducing the solution to that of a single array unit cell. The periodic boundary condition at unit cell side walls is enforced through a matrix transformation that mathematically "folds" opposing side walls onto each other with a phase shift appropriate to the array lattice and scan angle. The unit cell electric field is expanded in vector finite elements. Galerkin's method is used to cast the problem as a matrix equation, which is solved by the conjugate gradient method. A general-purpose computer code was developed and validated for cases of open-ended waveguides, microstrip patches, clad monopoles and printed flared notches, showing that the analysis method is accurate and versatile.
\end{abstract}

\section{INTRODUCTION}

QUCCESSFUL phased array element design has usually $\checkmark$ required the use of accurate numerical methods for predicting the scan-dependent gain and impedance [1], [2]. Recent attempts to develop phased arrays for broadband applications have led to radiator designs whose structures could not be adequately modeled with existing numerical methods. The need for a method that could account for complex feed structures, irregular conductor configurations and inhomogeneous dielectrics led to consideration of the finite element method (FEM), which has been used successfully for problems in electromagnetic scattering by using integral equations to impose radiation conditions [3]-[5]. These combinations of FEM with the method of moments (MoM) are usually referred to as hybrid finite element methods (HFEM).

This paper describes a hybrid finite element method for predicting the performance of radiators in an infinite phased array. The analysis considers the general case of one radiator in an infinite array, fed through a ground plane by a cylindrical waveguide. Accurate feed modeling is accomplished by enforcing continuity between the FEM solution and an arbitrary number of waveguide modes across the ground plane aperture. A periodic integral equation is imposed at a plane above the

Manuscript received November 1, 1993; revised June 2, 1994

D. T. McGrath is with U.S.A.F. Phillips Laboratory Kirtland AFB, NM 87117 USA.

V. P. Pyati is with the Air Force Institute of Technology Wright-Patterson AFB, OH 45433 USA.

IEEE Log Number 9407160 . antenna's physical structure to enforce the radiation condition and to confine the analysis to a single array unit cell. The electric field is expanded in terms of vector finite elements inside the unit cell, and Galerkin's method is used to write the problem as a matrix equation. The Floquet conditions on the unit cell side walls are enforced as transformations of the matrix that effectively "wrap" opposing unit cell walls onto each other with a phase shift appropriate to the scan angle and unit cell dimensions. The solution of the linear system gives a piecewise-linear approximation to the electric field, from which active reflection coefficient and active element gain are computed. Validation tests with a general-purpose computer code confirm that the method is versatile, accurate and reliable.

\section{FORMULATION}

\section{A. General Description}

Fig. 1 illustrates the generic problem geometry. The array is planar, periodic in both the $\mathrm{x}$ and $\mathrm{y}$ directions, and has an arbitrary skew angle, $\gamma$. The volume region $\Omega$ is a single unit cell truncated below at the waveguide aperture, and at a plane $z=h$ above the radiator's physical structure. The surface $\Gamma$ bounding $\Omega$ is the union of: a) all perfectly conducting surfaces; b) the "radiation boundary," $\Gamma_{R}$; c) the waveguide aperture, $\Gamma_{W}$; and d) the unit cell side walls $\Gamma_{x+}, \Gamma_{x-}, \Gamma_{y+}$, and $\Gamma_{y-} . \Omega$ may contain any number of material regions with distinct complex permittivity, $\epsilon$, and permeability, $\mu$, as well as any number of perfectly conducting obstacles, including infinitely thin wires and plates.

A numerical description of the problem geometry is created by subdividing $\Omega$ into small volume elements, or "cells." In this work, the cells are tetrahedra. The example exploded mesh shown in Fig. 2 is for a unit cell of a printed flared notch array. The cells in the center represent the dielectric substrate, while those on the left and right are free space. This radiator is one of the types that motivated this work because it includes an inhomogeneous dielectric and irregularly-shaped conductors.

\section{B. Expansion, Weighting and the Matrix Equation}

The electric field everywhere inside $\Omega$ and on its bounding surfaces is approximated by the following expansion:

$$
\bar{E} \approx \tilde{E}=\sum_{s=1}^{N} e_{s} \bar{\psi}_{s}(x, y, z)
$$

where each $e_{s}$ is a complex scalar and each $\overline{\psi_{s}}$ is a real-valued vector function. $N$ is the number of mesh edges that are not 


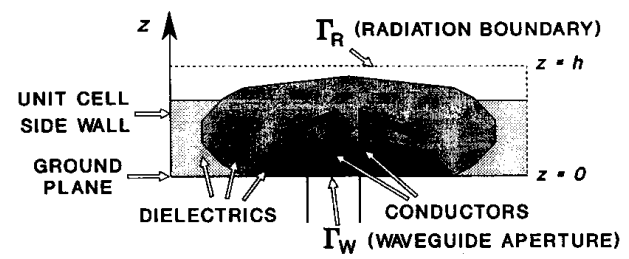

(a)

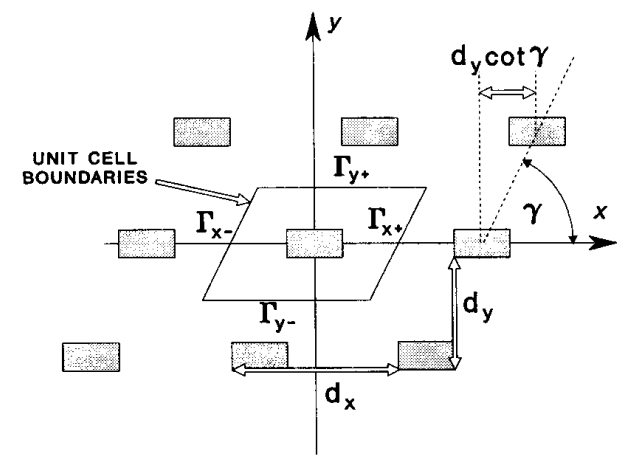

(b)

Fig. 1. Generic problem geometry: (a) unit cell cross section; and (b) array lattice.

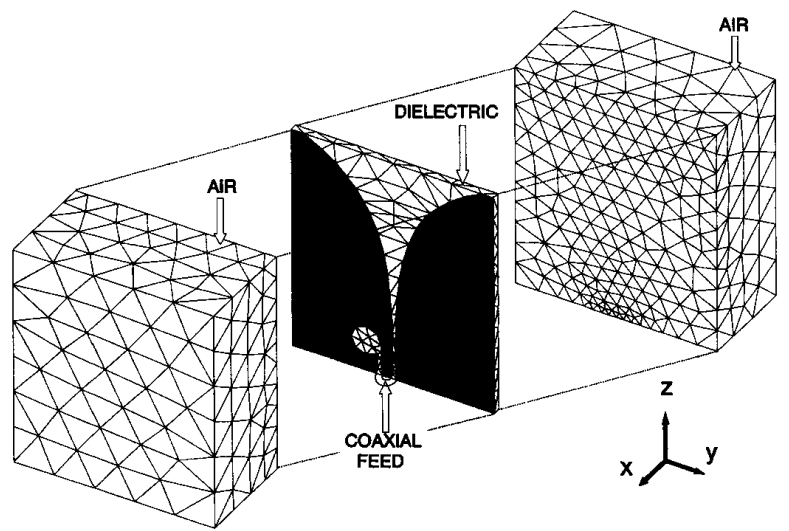

Fig. 2. Example unit cell finite element mesh: printed flared notch radiator with coaxial feed. Conducting surface is shaded.

on perfectly conducting surfaces. Each $\overline{\psi_{s}}$ is defined within the mesh cells adjacent to edge $s$, and is zero elsewhere. $\overline{\psi_{s}}$ is the "vector edge element" [4], [6]:

$$
\bar{\psi}_{s}=\ell_{i j}\left(f_{i} \nabla f_{j}-f_{j} \nabla f_{i}\right)
$$

where $i$ and $j$ are the indexes of the nodes bounding edge $s$, and $f_{i}$ and $f_{j}$ are the corresponding linear scalar node-based elements [7]. The scaling by the edge length $\ell_{i j}$ ensures that the component of $\overline{\psi_{s}}$ tangent to the edge is a unit vector. These expansion functions have two important advantages over scalar functions: a) they are divergenceless, so that the expansion (1) will satisfy the divergence condition; and b) they enforce continuity of the tangential field components across cell boundaries while allowing the normal component to be discontinuous, so that they correctly model dielectric discontinuities [6], [8].

The electric field must satisfy the source-free, timeharmonic vector wave equation

$$
L(\bar{E})=\nabla \times \frac{1}{\mu_{r}} \nabla \times \bar{E}-k_{0}^{2} \epsilon_{r} \bar{E}=0
$$

where $k_{0}$ is the free space wavenumber. This form allows $\epsilon_{r}$ and $\mu_{r}$ to be functions of position. The residual error due to the approximation (1) is $\bar{R}=L(\bar{E})-L(\tilde{E})$. The weighted residuals procedure sets $\langle\bar{R}, \bar{W}>=0$, where $\bar{W}$ is a weighting function and the inner product integration is taken over all of $\Omega$. Applying a Green's identity to the integral produces the "weak form":

$$
\begin{aligned}
F(\bar{E}) & =\int_{\Omega}\left[\frac{1}{\mu_{r}} \nabla \times \bar{W}^{*} \cdot \nabla \times \bar{E}-k_{0}^{2} \epsilon_{r} \bar{W}^{*} \cdot \bar{E}\right] d v \\
& -j k_{0} \eta_{0} \int_{\Gamma} \bar{W}^{*} \cdot(\hat{n} \times \bar{H}) d s=0
\end{aligned}
$$

where $\hat{n}$ is the outward normal to $\Omega$ and $\eta_{0}$ is the impedance of free space. The discretization of this functional using Galerkin's method generates a system of equations. That system is modified by a transformation $R$ representing side wall periodicity conditions, giving

$$
[R]\left[S^{I}+S^{W}+S^{R}\right][R]^{H} \boldsymbol{E}=\boldsymbol{E}^{\text {inc }} .
$$

(Superscript $\mathrm{H}$ denotes Hermitian, or conjugate transpose.) The matrix $S^{I}$ originates in the volume integral in (4), while $S^{W}$ and $S^{R}$ originate in the surface integral taken over $\Gamma_{W}$ and $\Gamma_{R}$, respectively. The right side column vector $\boldsymbol{E}^{\text {inc }}$ results from integrating the incident field term over $\Gamma_{W}$. Finally, the solution vector $\boldsymbol{E}$ gives the coefficients $e_{s}$ in (1).

\section{Interior Discretization}

Initially, the unit cell mesh is treated as though all nonconducting portions of $\Gamma$ are open-circuit walls, where the mesh is simply truncated without regard to boundary conditions. When the expansion (1) is substituted into (4), it gives one equation in $N$ unknowns. Using Galerkin's method, each $\overline{\psi_{t}}$ is substituted in turn for $\bar{W}$, giving $N$ equations. The result is that the terms of $S^{I}$ are given by the volume integrals

$$
S_{s t}^{I}=\int_{\Omega_{s t}}\left[\mu_{r}^{-1} \nabla \times \overline{\psi_{s}} \cdot \nabla \times \overline{\psi_{t}}-k_{0}^{2} \epsilon_{r} \overline{\psi_{s}} \cdot \overline{\psi_{t}}\right] d v .
$$

The domain of integration, $\Omega_{s t}$, is the union of all cells shared by edges $s$ and $t$.

\section{Waveguide Integral Equation}

Satisfying field continuity across $\Gamma_{W}$ requires an integral equation for tangential $\bar{H}$ in terms of $\bar{E}$ on $\Gamma$. This is obtained in a manner similar to that used by Harrington and Mautz to 
solve for waveguide radiation with the method of moments [9], starting with a modal expansion from Marcuvitz [10]:

$$
\bar{E}^{t}=e^{-\gamma_{\nu} z} \bar{g}_{\nu}+\sum_{i=0}^{\infty} C_{i} e^{\gamma_{i} z} \bar{g}_{i}
$$

The superscript $t$ denotes transverse (to $z$ ), $\overline{g_{i}}$ is an orthonormal mode function with propagation constant $\gamma_{i}$, and the index $i$ includes all $\mathrm{TE}_{m n}$ and $\mathrm{TM}_{m n}$ modes. The first term is due to a unit-amplitude incident field in the mode $\nu$, usually the dominant mode. The unknown coefficients $C_{i}$ may be found from the transverse electric field over $\Gamma_{W}$ as

$$
C_{i}=\int_{\Gamma_{W}} \bar{E}^{t} \cdot \bar{g}_{i} d s-\delta_{\nu i}
$$

where $\delta_{\nu i}$ is a Kronecker delta. $C_{0}$ is the reflection coefficient for the dominant waveguide mode. The transverse magnetic field is given in terms of the modal admittances $Y_{i}$ :

$$
\bar{H}^{t}=Y_{\nu} e^{-\gamma_{\nu} z}\left(\hat{z} \times \bar{g}_{\nu}\right)-\sum_{i=0}^{\infty} C_{i} Y_{i} e^{\gamma_{i} z}\left(\hat{z} \times \bar{g}_{i}\right)
$$

Substituting (8) into (9) gives $\overline{H^{t}}$ in terms of $\overline{E^{t}}$ on $\Gamma_{W}$. Then evaluating $\hat{n} \times \overline{H^{t}}$ on $\Gamma_{W}(\hat{n}=\hat{z})$ gives the integral equation

$$
\hat{n} \times \bar{H}^{t} \underset{\Gamma_{W}}{\mid}=2 Y_{\nu} \bar{g}_{\nu}-\sum_{i=0}^{\infty} Y_{i} \bar{g}_{i} \int_{\Gamma_{W}} \bar{E}^{t} \cdot \bar{g}_{i} d s .
$$

The result of substituting the right side into (4), followed by expansion and testing is

$$
\begin{gathered}
S_{s t}^{W}=j k_{0} \eta_{0} \sum_{i=0}^{\infty} Y_{i} \Psi_{s i} \Psi_{t i}, \quad s, t \in \Gamma_{W} \\
\Psi_{s i}=\int_{\Gamma_{W}} \bar{\psi}_{s} \cdot \bar{g}_{i} d s \\
E_{t}^{i n c}=2 j k_{0} \eta_{0} Y_{\nu} \Psi_{t \nu}, \quad t \in \Gamma_{W} .
\end{gathered}
$$

The inner products of expansion functions and waveguide mode functions may be carried out in closed form for rectangular waveguide; or numerically for circular and circular coaxial waveguide [12].

\section{E. Periodic Integral Equation}

Each radiator in the infinite array is assumed to be excited by a unit-amplitude incident field in the waveguide from $z$ $<0$. The excitation phases produce a beam directed toward $\theta_{0}, \phi_{0}$ in spherical coordinates. The fields above $z=0$ must obey the condition

$$
\begin{gathered}
\bar{E}\left(x+m d_{x}+n d_{y} \cot \gamma, y+n d_{y}\right) \\
=\bar{E}(x, y) e^{-j \beta_{x}\left(m d_{x}+n d_{y} \cot \gamma\right)} e^{-j \beta_{y} n d_{y}} \\
\beta_{x}=k_{0} \sin \theta_{0} \cos \phi_{0} \\
\beta_{y}=k_{0} \sin \theta_{0} \sin \phi_{0} .
\end{gathered}
$$

An integral equation for the magnetic field transverse to $\Gamma_{R}$ in terms of the electric field transverse to $\Gamma_{R}$ is

$$
\begin{gathered}
-\hat{n} \times \bar{H}\left(\Gamma_{R}\right) \\
=\sum_{m=-\infty}^{\infty} \sum_{n=-\infty}^{\infty} \stackrel{\leftrightarrow}{T}_{m n} \cdot \underline{E}_{u c}\left(k_{x m n}, k_{y m n}\right) e^{-j k_{x m n} x} e^{-j k_{y m n} y} \\
k_{x m n}=k_{0} \sin \theta_{0} \cos \phi_{0}-\frac{2 \pi m}{d_{x}} \\
k_{y m n}=k_{0} \sin \theta_{0} \sin \phi_{0}-\frac{2 \pi n}{d_{y}}+\frac{2 \pi m \cot \gamma}{d_{x}} \\
\overleftrightarrow{T}_{m n}=\frac{\left[\begin{array}{c}
k_{0}^{2}-k_{x m n}^{2}-k_{y m n}^{2} \\
d_{x} d_{y} k_{0} \eta_{0}
\end{array}\right.}{\cdot\left[\begin{array}{cc}
\left(k_{0}^{2}-k_{y m n}^{2}\right) & k_{x m n} k_{y m n} \\
k_{x m n} k_{y m n} & \left(k_{0}^{2}-k_{x m n}^{2}\right)
\end{array}\right]}
\end{gathered}
$$

where the negative imaginary result is chosen when the square root is negative, and $\underline{\underline{E}}_{u c}\left(k_{x}, k_{y}\right)$ is the two-dimensional Fourier transform of the unit cell electric field transverse to $\Gamma_{R}$ :

$$
\begin{aligned}
\underline{E}_{u c} & =\sum_{s=1}^{N} e_{s} \bar{\xi}_{s}\left(k_{x}, k_{y}\right) \\
& =\sum_{s=1}^{N} e_{s} \int_{\Gamma_{R}} \bar{\psi}_{s} e^{j k_{x} x} e^{j k_{y} y} d x d y .
\end{aligned}
$$

Substituting this expression into the surface integral of (4), then testing with each $\psi_{t}$ gives

$$
\begin{aligned}
S_{s t}^{R}= & j k_{0} \eta_{0} \sum_{m} \sum_{n} \bar{\xi}_{s m n} \cdot \stackrel{\leftrightarrow}{T}_{m n} \\
& \cdot \int_{\Gamma_{R}} \bar{\psi}_{t} e^{-j k_{x m n} x} e^{-j k_{y m n} y} d x d y, \quad s, t \in \Gamma_{R}
\end{aligned}
$$

where $\bar{\xi}_{s m n}$ denotes $\bar{\xi}_{s}\left(k_{x m n}, k_{y m n}\right)$. The integral in (22) is equivalent to $\bar{\xi}_{t m n}^{*}\left(\bar{\psi}_{t}\right.$ is a real function, therefore its Fourier transform is Hermitian [13:193], i.e., $\overline{\xi_{t}}\left(-k_{x},-k_{y}\right)=$ $\left.\bar{\xi}_{t}^{*}\left(k_{x}, k_{y}\right)\right)$, so the final expression for the entries in the matrix $S^{R}$ is

$$
S_{s t}^{R}=j k_{0} \eta_{0} \sum_{m} \sum_{n} \bar{\xi}_{s m n} \cdot \stackrel{\leftrightarrow}{T}_{m n} \cdot \bar{\xi}_{t m n}^{*}, \quad s, t \in \Gamma_{R}
$$

The edges that are on both $\Gamma_{R}$ and a unit cell boundary are treated differently. There are no entries in $S^{R}$ for edges on $\Gamma_{x+}$ and $\Gamma_{y+}$. Instead, the entries for edges on $\Gamma_{x-}$ and $\Gamma_{y-}$ are computed as if the mesh extended into the adjacent unit cells. This is consistent with Gedney's concept of "overlap elements" [14]. It ensures that there is no conflict between the periodic radiation condition and the side-wall periodicity conditions. 


\section{F. Side-Wall Periodicity Conditions}

The mesh is assumed to have been created so that the surface mesh on opposing side walls is identical. Then each mesh edge on $\Gamma_{x+}$ or $\Gamma_{y+}$ has an "image edge" (the term does not relate to electric or magnetic images) on the opposing boundary that is identical except for a translation of $\left(-d_{x}, 0\right)$ or $\left(-d_{y} \cot \gamma,-d_{y}\right)$, respectively. The implementation of Floquet's condition on these boundaries may be viewed conceptually as follows: The $\Gamma_{x+}$ and $\Gamma_{y+}$ edges will be merged with their $-x$ and $-y$ counterparts, effectively re-creating an infinite mesh by wrapping opposing side walls onto each other. Suppose, for example, that edge \#19 is on $\Gamma_{x+}$ and its image edge on $\Gamma_{x-}$ is edge \#2. The original matrix will have a pair of entries $S_{19, k}$ and $S_{k, 19}$ for every edge $k$ that shares at least one cell with edge \#19. In transforming the matrix, these entries will be eliminated and new ones added. The new entries are: $S_{2, k}^{\prime}$ $=S_{19, k} \exp \left\{j \psi_{x} d_{x}\right\}$ and $S_{k, 2}^{\prime}=S_{k, 19} \exp \left\{-j \psi_{x} d_{x}\right\}$ for edges $k$ that are not also on $\Gamma_{x+}$; and $S_{2, j}^{\prime}=S_{2, j}+S_{19, k}$ and $S_{j, 2}^{\prime}$ $=S_{j, 2}+S_{k, 19}$ for each edge $k$ on $\Gamma_{x+}$ whose image edge is edge $j . \Gamma_{y+}$ is handled similarly, except that the phase shift terms are $\exp \left\{ \pm \mathrm{j}\left(\psi_{x} d_{y} \cot \gamma+\psi_{y} d_{y}\right)\right\}$.

This procedure may be regarded as the transformation

$$
S^{\prime}=R S R^{H} \text {. }
$$

The matrix $R$ is $M \times N$, where $N-M$ is the number of edges on $\Gamma_{x+}$ and $\Gamma_{y+}$ combined. Its first $M$ columns are the $M \times M$ identity matrix. In the remaining columns, there is an entry $R_{i k}$ for each pair of image edges:

$$
\begin{gathered}
R_{i k}= \\
\left\{\begin{array}{cc}
e^{j \beta_{x} d_{x}}, & k \in \Gamma_{x+}, i \in \Gamma_{x-} \\
e^{j\left(\beta_{x} d_{y} \cot \gamma+\beta_{y} d_{y}\right)}, & k \in \Gamma_{y+}, i \in \Gamma_{y-} \\
e^{j\left(\beta_{x} d_{x}+\beta_{y} d_{y} \cot \gamma+\beta_{y} d_{y}\right)}, & k \in \Gamma_{x+} \cap \Gamma_{y+}, i \in \Gamma_{x-} \cap \Gamma_{y-}
\end{array}\right.
\end{gathered}
$$

This assumes an ordering of edges so that those in $\Gamma_{x+}$ and $\Gamma_{y+}$ are last. The transformation does not affect $S^{W}$ or $S^{R}$ because those matrices do not have any nonzero entries for edges in $\Gamma_{x+}$ or $\Gamma_{y+}$.

\section{VALIDATION AND DEMONSTRATION}

The preceding theory was implemented in a general-purpose computer code. The code is geometry-independent, owing to the use of commercial software for geometry definition and mesh generation. It uses the conjugate gradient method (CGM) [15] to solve the system of equations. The example results that follow are both validation (comparisons to solutions obtained by other methods) and demonstration (calculations for problems that have not been addressed by other methods). In all cases, the incident field was in the dominant waveguide mode and 32 waveguide modes $(0 \leq m, n \leq 3)$ and 121 Floquet modes $(-5 \leq \mathrm{m}, \mathrm{n} \leq 5)$ were included in calculating $S^{W}$ and $S^{R}$, respectively.

\section{A. Waveguide Arrays}

Arrays of open-ended waveguides, horns, and slots are a simplified case of the preceding analysis [16]. These cavity-

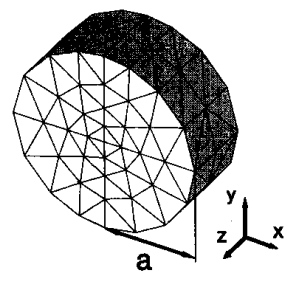

(a)

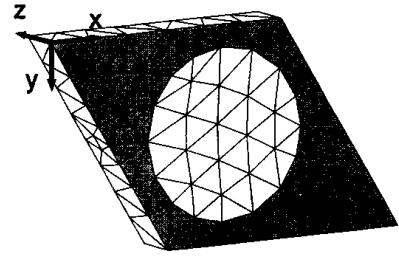

(b)
Fig. 3. Two circular waveguide models: (a) waveguide section below ground plane; and (b) unit cell section above ground plane.

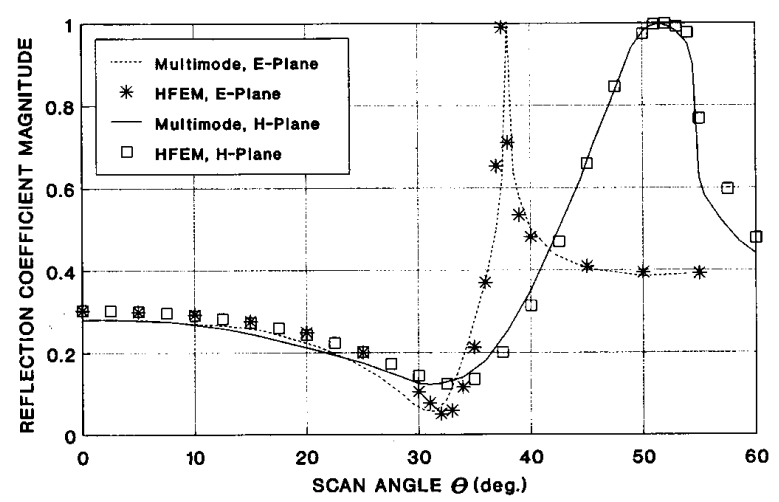

Fig. 4. Comparison of multimode [2] and hybrid finite element calculations of active reflection coefficient magnitude: $d_{x}=0.714 \lambda_{0}, d_{y}=0.619 \lambda_{0}$, $a=0.343 \lambda_{0}$ (waveguide radius), $\gamma=60^{\circ}$.

type elements couple only through apertures in a common, conducting ground plane. Hence, the formulation may be simplified by excluding the matrix transformation (24). Fig. 3 shows two alternative representations of a unit cell for a circular waveguide. In Fig. 3(a), the mesh represents a section of waveguide under the ground plane, while Fig. 3(b) is a section of unit cell above the ground plane. In each case the cell faces bordering conductors are shaded.

Fig. 4 compares Amitay's multimode calculations [2:276] with HFEM using the model from Fig. 3(a). This close agreement establishes the validity of the waveguide and periodic radiation conditions. Similar agreement was obtained for circular waveguides with dielectric plugs, rectangular waveguides, and pyramidal horns [11], [16]. Fig. 5 compares HFEM calculations for both models in Fig. 3 (different lattice spacing than the Fig. 4 calculations). The agreement between these two validates the side wall periodicity conditions.

\section{B. Microstrip Arrays}

Two cases of rectangular microstrip patch arrays on highpermittivity substrates $\left(\epsilon_{r}=12.8\right.$, representing Gallium Arsenide) were modeled by Pozar and Schaubert [17] using the method of moments to demonstrate surface wave effects. The two substrate thicknesses were $h=0.02 \lambda_{0}$ (thin case) and $h=0.06 \lambda_{0}$ (thick case). In the finite element model, the patch feed pin is an extension of the coaxial feed's center conductor. 


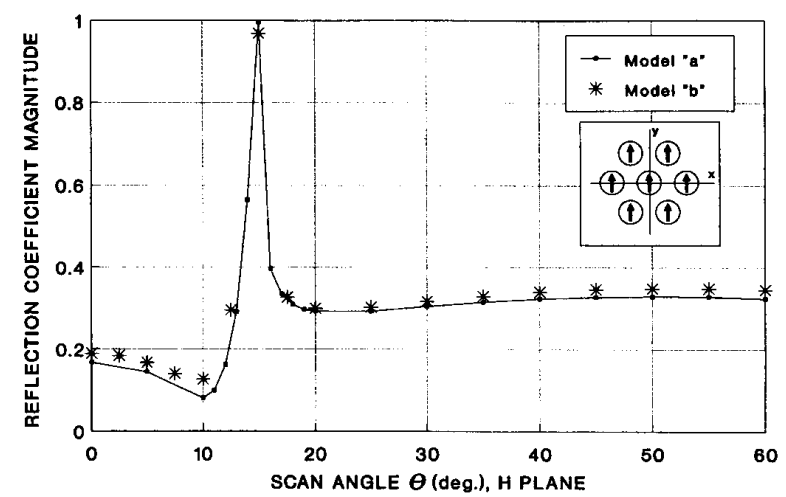

Fig. 5. Comparison of HFEM using alternate circular waveguide array models (Fig. 3(a) and (b): $d_{x}=0.92 \lambda_{0}, d_{y}=0.8 \lambda_{0}, a=0.32 \lambda_{0}, \gamma=60^{\circ}$.

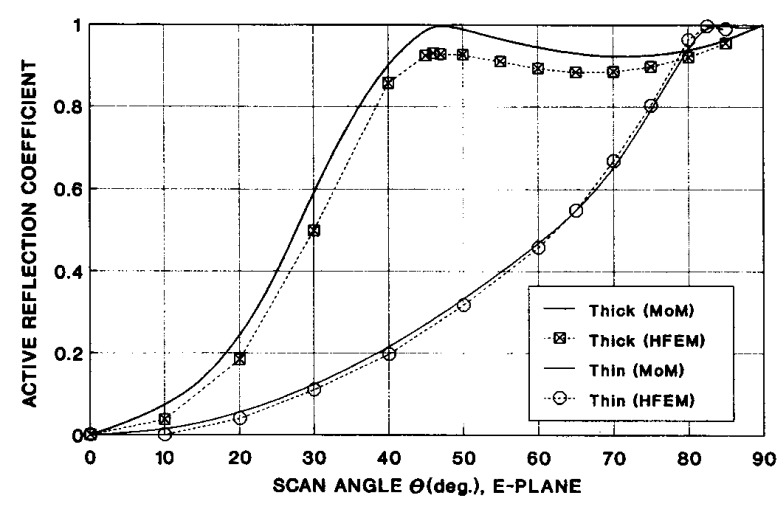

Fig. 6. Comparison of moment method [17] and HFEM calculations of active reflection coefficient for two microstrip patch arrays with $d_{x}=0.5 \lambda_{0}, d_{y}=0.5 \lambda_{0}$ and $\epsilon_{r}=12.8$. Thin Case: $h=0.02 \lambda_{0}$, width $=0.15 \lambda_{0}$ and length $=0.131 \lambda_{0}$; Thick Case: $h=0.06 \lambda_{0}$, width $=0.15 \lambda_{0}$ and length $=0.098 \lambda_{0}$.

Fig. 6 is a comparison of the MoM calculations and the HFEM calculations. It shows the normalized active reflection coefficient, emulating the effect of a matching network that adjusts for zero reflection coefficient at broadside scan:

$$
R_{\text {norm }}=\left|\frac{Z_{\text {in }}(\theta, \phi)-Z_{i n}(0,0)}{Z_{\text {in }}(\theta, \phi)+Z_{\text {in }}(0,0)}\right| \text {. }
$$

Both MoM and HFEM are predicting the existence and approximate angular location of blindnesses due to surface waves in the dielectric slabs. The slight disagreement for the thick case is due to a difference in the feed model: while the MoM used an idealized probe, the HFEM used a coaxial aperture (inner and outer radii of $0.0075 \lambda_{0}$ and $0.0175 \lambda_{0}$, respectively). Aberle and Pozar [18] showed that when an accurate coaxial feed model is included, the MoM calculations also give a reflection coefficient that is slightly below 1.0 at the blindness angle.

\section{Printed Flared Notch Array}

The final test case, a printed flared notch, is representative of the initial motivation for this work. The design shown in Fig.

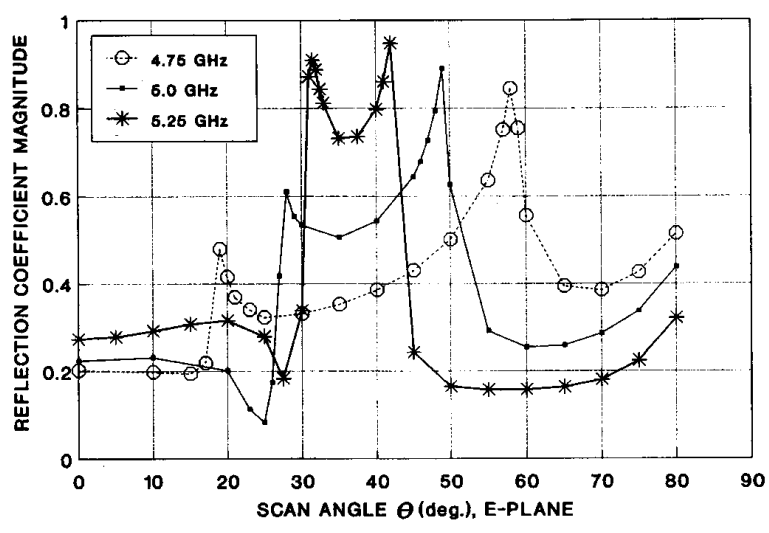

(a)

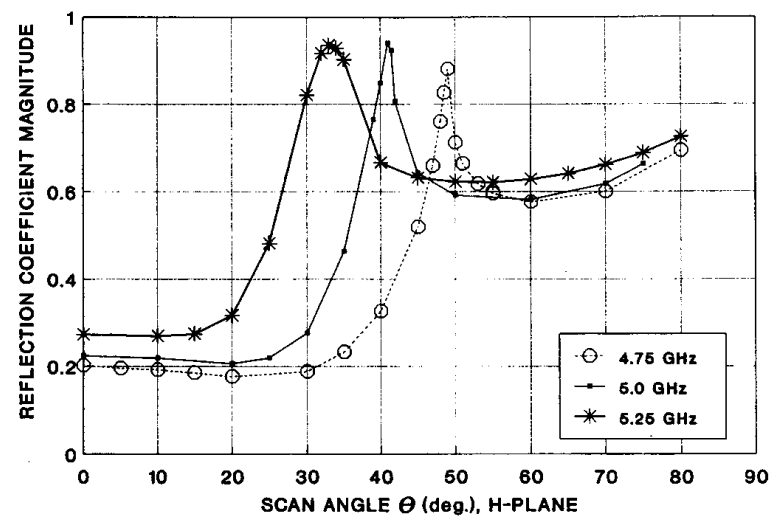

(b)

Fig. 7. HFEM predictions of active reflection coefficient versus scan angle for printed flared notch (Fig. 2): $d_{x}=36 \mathrm{~mm}, d_{y}=34 \mathrm{~mm}$, rectangular lattice, substrate thickness $=1.27 \mathrm{~mm}$ with $\epsilon_{r}=6$, flare length $=33.3 \mathrm{~mm}$, overall length= $38.1 \mathrm{~mm}$ : (a) E-plane scans; (b) H-plane scans.

2 uses a novel coplanar waveguide (CPW)-slotline transition by Ho and Hart [19] to restrict metallization to one side of the substrate only. The coaxial waveguide feeds a short section of coplanar waveguide that transitions to slotline by terminating one of the parallel slots in a broadband open. The slotline then leads into an exponential flare [20]. Note that since the substrate has a much higher permittivity $\left(\epsilon_{r}=6\right)$ than the surrounding air, the mesh is much finer in the substrate, and gradually relaxes going out towards the $+x$ and $-x$ unit cell side walls. The mesh is made even finer in the vicinity of the coax and CPW-slotline transition in order to accurately capture the details of the geometry.

The HFEM predictions for active reflection coefficient versus scan angle are shown in Figs. 7(a) and (b). The radiator is well-matched to the array environment, with reflection coefficient less than 0.3 from 3.0 to $5.0 \mathrm{GHz}$. Scan blindnesses occur at the grating lobe angle in both $\mathrm{E}$ and $\mathrm{H}$ planes when the frequency exceeds that at which the inter-element spacing is more than one half wavelength $(4.4 \mathrm{GHz}$ in the E plane; $4.2 \mathrm{GHz}$ in the $\mathrm{H}$ plane). There is an additional $\mathrm{E}$ 
plane blindness that moves outward in angle with increasing frequency. Schaubert and Aas have shown that this unusual behavior is due to the excitation of a waveguide mode on the corrugated structure [21].

\section{CONCLUSIONS}

The finite element method was applied to the analysis of phased array radiators. This required developing boundary conditions for waveguide feeds, a periodic radiation condition, and side wall periodicity conditions. The first of these provides an accurate means of modeling rectangular, circular and coaxial waveguide feeds. The second and third allow accurate modeling of radiation from elements in large (assumed infinite) arrays, including scan blindness effects. The validation and demonstration cases showed that the techniques are valid for radiators with complex feed structures, irregular conductor configurations and inhomogeneous dielectrics. Finally, the fact that the same computer code was used for all of these cases demonstrates the unprecedented versatility obtainable using the hybrid finite element method.

\section{ACKNOWLEDGMENT}

The authors are indebted to Professors W. Baker, M. Mehalic, D. Quinn and P. Skinner of the Air Force Institute of Technology for their advice and constructive criticism throughout the course of this work. They also thank Dr. R. Mailloux of the Air Force Rome Laboratory for his encouragement and assistance.

\section{REFERENCES}

[1] L. Stark, "Microwave theory of phased-array antennas-A review," Proc. IEEE, vol. 62, pp. 1661-1701, Dec. 1974.

[2] N. Amitay, V. Galindo, and C. Wu, Theory and Analysis of Phased Array Antennas. New York: Wiley, 1972.

[3] J-M. Jin and V. V. Liepa, "Application of the hybrid finite element method to electromagnetic scattering from coated cylinders," IEEE Trans. Antennas Propagat., vol. AP-36, pp. 50-54, Jan. 1988.

[4] J. D. D'Angelo and I. D. Mayergoyz, "Finite element methods for the solution of RF radiation and scattering problems," Electromagnetics, vol. 10 , pp. 177-199, 1990.

[5] W. E. Boyse and A. A. Seidl, "A hybrid finite element and moment method for electromagnetic scattering from inhomogeneous objects," in 7th Annu. Rev. Progress in Applied Computational Electromagnetics, Naval Postgraduate School, Monterey, CA, Mar. 1991, pp. 160-169.

[6] M. L. Barton, and Z. J. Cendes, "New vector finite elements for threedimensional magnetic field computation," J. Appl. Phys., vol. 61, pp. 3919-3921, Apr. 1987.

[7] P. P. Silvester and R. L. Ferrari, Finite Elements for Electrical Engineers, 2nd ed. Cambridge, MA: Cambridge Univ. Press, 1990

[8] G. Mur, "Finite-element modeling of three-dimensional electromagnetic fields in inhomogeneous media," Radio Sci., vol. 26, pp. 275-280, 1991.
[9] R. F. Harrington and J. R. Mautz, "A generalized network formulation for aperture problems," IEEE Trans. Antennas Propagat., vol. AP-24, pp. 870-873, Nov. 1976.

[10] N. Marcuvitz, Waveguide Handbook. New York: McGraw-Hill, 1951.

[11] D. T. McGrath, "Phased array antenna analysis using hybrid finite element methods," Ph.D. dissertation, Air Force Institute of Technology, Wright-Patterson AFB, OH, June 1993.

[12] _ "Hybrid finite element/waveguide mode analysis of passive RF devices," Rome Laboratory, Griffiss AFB, NY, RL-TR-93-130, June 1993.

[13] J. D. Gaskill, Linear Systems, Fourier Transforms, and Optics. New York: Wiley, 1978

[14] S. D. Gedney, J. F. Lee, and R. Mittra, "A combined FEM/MoM approach to analyze the plane wave diffraction by arbitrary gratings," IEEE Trans. Microwave Theory Tech., vol. 40, pp. 363-370, Feb. 1992.

[15] T. K. Sarkar and E. Arvas, "On a class of finite step iterative methods (conjugate directions) for the solution of an operator equation arising in electromagnetics," IEEE Trans. Antennas Propagat., vol. AP-33, pp. 1058-1066, Oct. 1985.

[16] D. T. McGrath and V.P. Pyati, "Hybrid finite element/waveguide mode analysis for infinite phased arrays of cavity radiators," in 1993 IEEE AP-S Symp. Dig., June 1993, pp. 1242-1245.

[17] D. M. Pozar and D. H. Schaubert, "Analysis of an infinite array of rectangular microstrip patches with idealized probe feeds," IEEE Trans. Antennas Propagat., vol. AP-32, pp. 1101-1107, Oct. 1984.

[18] J. T. Aberle and D. M. Pozar, "Analysis of infinite arrays of one- and two-probe-fed circular patches," IEEE Trans. Antennas Propagat., pp. 421-432, Apr. 1990.

[19] T. Q. Ho and S. M. Hart, "A broad-band coplanar waveguide to slotline transition," IEEE Microwave and Guided Wave Lett., vol. 2, pp. 415-416, Oct. 1992.

[20] Y. H. Choung and C. C. Chen, "44 GHz slotline phased array antenna," in 1989 IEEE AP-S Symp. Dig., June 1989, pp. 1730-1733.

[21] D. H. Schaubert and J. A. Aas, "An explanation of some E-plane scan blindnesses in single-polarized tapered slot arrays," in 1993 IEEE AP-S Symp. Dig., June 1993, pp. 1612-1615.

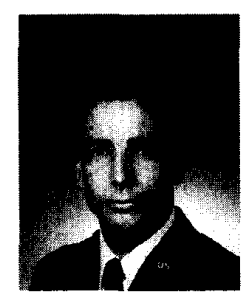

Daniel T. McGrath (M'94) received the B.S.E.E degree from the U.S.A.F. Academy in 1979 and the M.S.E.E. and Ph.D. degrees from the Air Force Institute of Technology, Wright-Patterson AFB, OH, in 1982 and 1993, respectively.

He worked at the Air Force Armament Laboratory, Eglin AFB, FL (1979-1981), in the area of signal processing and pattern recognition for smart munitions, and at the Rome Air Development Center, Hanscom AFB, MA (1983-1987) in phased array and lens antennas for radar and telecommunications. He authored two U.S. patents on constrained lens antenna designs. He was also a coauthor of the 1987 AP-S Wheeler Applications Prize Paper. $\mathrm{He}$ is presently assigned to the U.S.A.F. Phillips Laboratory, Kirtland AFB, $\mathrm{NM}$, participating in high power microwave and transient electromagnetics research.

Vittal P. Pyati, photograph and biography not available at the time of publication. 